\title{
Tunnel magnetoresistance in GaMnAs: Going beyond Jullière formula
}

\author{
L. Brey ${ }^{a)}$ \\ Instituto de Ciencia de Materiales de Madrid (CSIC), Cantoblanco, 28049, Madrid, Spain \\ C. Tejedor \\ Departmento de Física Teórica de la Materia Condensada, Universidad Autónoma de Madrid, \\ 28049 Madrid, Spain \\ J. Fernández-Rossier \\ Departamento de Física Aplicada, Universidad de Alicante, 03080 Alicante, Spain
}

(Received 17 May 2004; accepted 6 July 2004)

\begin{abstract}
The relation between tunnel magnetoresistance (TMR) and spin polarization is explored for GaMnAs/GaAlAs/GaMnAs structures where the carriers experience strong spin-orbit interactions. TMR is calculated using the Landauer approach. The materials are described in the 6 band $\mathbf{k} \cdot \mathbf{p}$ model which includes spin-orbit interaction. Ferromagnetism is described in the virtual crystal mean field approximations. Our results indicate that TMR is a function of spin polarization and barrier thickness. As a result of the stong spin-orbit interactions, TMR also depends on the the angle between current flow direction and the electrode magnetization. These results compromise the validity of Julliere formula. (C) 2004 American Institute of Physics. [DOI: 10.1063/1.1789241]
\end{abstract}

The relative orientation of the magnetization of two ferromagnetic electrodes can affect dramatically electron transport across a tunneling barrier connecting them. This phenomenon gives, rise to the so-called tunnel-magnetoresistance $^{1}$ (TMR), $\mathrm{TMR}=\left(R_{A P}-R_{P}\right) / R_{A P}$ where $R_{A P}$ and $R_{P}$ are the resistances for antiparallel and parallel orientations, respectively. TMR is exploited to fabricate devices which are ultrasensitive to variations of an external magnetic field. ${ }^{2}$ Microscopic understanding of TMR, based upon the hypothesis that spin is conserved in the tunneling process, leads to the well known Julliere formula: ${ }^{3,4}$

$$
\mathrm{TMR}^{J}=\frac{2 P_{L} P_{R}}{1+P_{L} P_{R}}
$$

which relates TMR with $P_{L, R}$, the polarizations of the left $(L)$ and right $(R)$ electrodes. Assuming Eq. (1) is correct, it permits extraction of the spin polarization of the electrodes from the value of TMR in symmetric tunnel junctions, regardless of the physical properties of the barrier. Equation (1) can be derived doing second order perturbation theory in tunneling amplitude. 5

Diluted magnetic semiconductors like $\mathrm{Ga}_{1-x} \mathrm{Mn}_{x} \mathrm{As}$ are ferromagnetic below $T_{C} \simeq 150 \mathrm{~K}$. ${ }^{6}$ This type of material raises much interest because they afford the integration of ferromagnetic and semiconducting functionalities in a single device. ${ }^{7,8}$ Substitutional impurities of $\mathrm{Mn}$ in GaAs are acceptors. The holes released by the $\mathrm{Mn}$ are responsible of the magnetic ordering and transport properties of $\mathrm{Ga}_{1-x} \mathrm{Mn}_{x} \mathrm{As}$. Several groups have been able to fabricate tunnel junctions with GaMnAs in the electrodes ${ }^{9-11}$ and they have reported large values of TMR finding that its value depends on the properties of the barrier. ${ }^{9}$ Furthermore, spin orbit (SO) interactions for the holes in the valence band of GaAs is very strong $\left(\Delta_{\mathrm{SO}} \simeq 0.34 \mathrm{eV}\right)$ so that the spin of the holes is not a good quantum number. Both the experimental results ${ }^{9-12}$ and the failure of Julliere's hypothesis on spin conservation, lead

${ }^{\text {a)Electronic mail: brey@icmm.csic.es }}$ us to study TMR in GaMnAs/AlAs/GaMnAs systems using a nonpertubative approach which fully includes spin-orbit interactions. We anticipate our main result: in GaMnAs based heterostructures, TMR depends on both the barrier thickness, $d_{b}$, the angle formed by the current flow and the magnetization. All of these features depend on the strength of the SO coupling and are relevant for the use of GaMnAs based heterostructures in magnetoelectronics.

In this letter we calculate vertical transport ( $z$ direction) in epitaxially grown GaMnAs/GaAlAs/GaMnAs tunnel systems. We take the same Mn density at the two electrodes. The materials are described in the 6 bands $\mathbf{k} \cdot \mathbf{p}$ model which captures the interplay of SO interaction and the threefold orbital degeneracy on the top of the valence bands. Ferromagnetism is originated by the exchange interaction between the spin of the localized Mn and the itinerant holes. In the case of metallic samples the mean field and virtual crystal (MF-VC) approximation ${ }^{13-16}$ accounts for a number of experimental facts like the dependence of $T_{c}$ on both the Mn and hole densities, ${ }^{13}$ the magnetic anisotropy ${ }^{14}$ and magnetic circular dichroism. ${ }^{13}$ In this approach spontaneous magnetization is characterized by effective Zeeman magnetic field $\mathcal{H}$ which represents the effect of exchange interaction of the Mn magnetization on the spin of the holes. The MF-VC approximation restores the translational invariance in the Hamiltonian, and it is possible to label the eigenvectors and eigenvalues by a band index, $n$, and a wave vector $\left(k_{z}, \mathbf{k}_{\|}\right)$. Due to the SO interaction, energy bands and Bloch states depend on both the magnitude and the orientation of $\mathcal{H}$, and spin is not conserved. For a given set of parameters representing GaMnAs, a magnetic orientation $\boldsymbol{\Omega}$ and a temperature, the above approach yields the carrier spin polarization $P$. In this model, the electronic structure of GaMnAs is fully characterized by $P$, the hole density $p$ and $\boldsymbol{\Omega}$. For a fixed Mn -hole exchange coupling strength, $p$ and $\boldsymbol{\Omega}$ there is a one to one correspondence between the Mn concentration and $P$. We describe the valence bands of the barrier (GaAlAs) with the same parameters used for GaAs, but offset ${ }^{17-19}$ a potential $V_{b}$ of the order of $300 \mathrm{meV}$. 


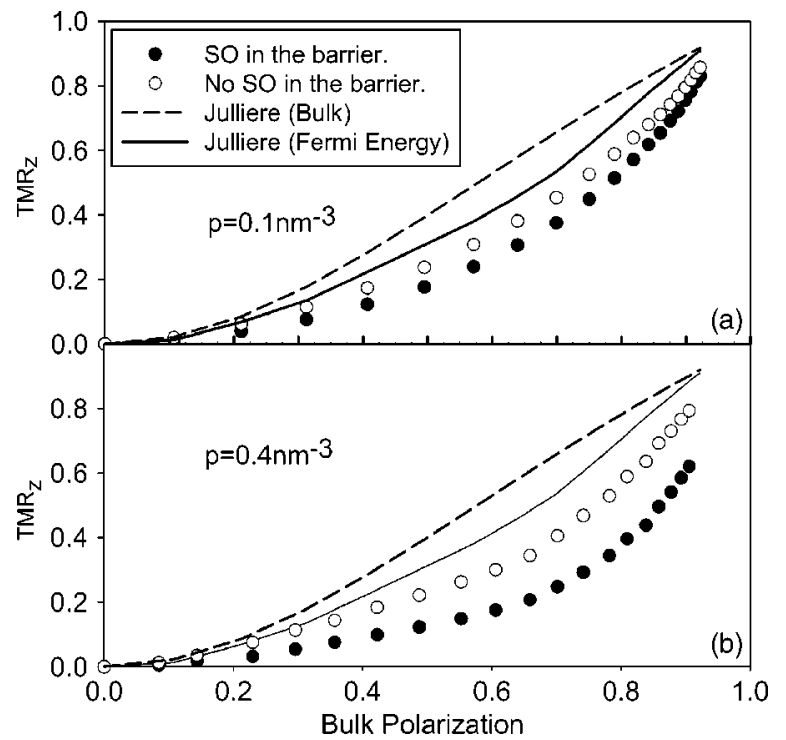

FIG. 1. TMR $z$ as function of $P d_{b}=15 \AA$ and $V_{b}=300 \mathrm{meV}$. Case (a) $p$ $=0.1 \mathrm{~nm}^{-3}$ and (b) $p=0.4 \mathrm{~nm}^{-3}$.

The conductance is calculated using the Landauer formula: ${ }^{17-19}$

$$
G_{\boldsymbol{\Omega}_{L}, \boldsymbol{\Omega}_{R}}=\frac{e^{2}}{2 \pi \hbar} \sum_{n, n^{\prime}, \mathbf{k}_{\|}} T_{n, n^{\prime}}^{\mathbf{k}_{\|}}\left(E_{F}\right),
$$

where $E_{F}$ is the Fermi energy, $T_{n, n}^{\mathbf{k}_{\|}}\left(E_{F}\right)$ is the transmission probability from a state on the left with band index $n$ to a state on the right with band index $n^{\prime}$, and $\boldsymbol{\Omega}_{L, R}$ are the magnetization orientation of the electrodes. Only the energy and $\mathbf{k}_{\|}$are conserved in the tunneling process. The transmission matrix is obtained in the transfer matrix method applied to the 6 bands $\mathbf{k} \cdot \mathbf{p}$ Hamiltonian ${ }^{17-19}$ including the exchange field $\mathcal{H}^{20}$

Conventionally, $G$ depends on the angle formed by $\boldsymbol{\Omega}_{L}$ and $\boldsymbol{\Omega}_{R}$. In the presence of SO coupling the conductance depends also on the angle formed by the current with the magnetization. This makes it necessary to define both $\mathrm{TMR}_{z}$, for the case of current parallel (or antiparallel) with the magnetization, and $\mathrm{TMR}_{x}$, for the case where the magnetizations are perpendicular to the current flow:

$$
\mathrm{TMR}_{z}=\frac{G_{\uparrow, \uparrow}-G_{\uparrow, \downarrow}}{G_{\uparrow, \uparrow}}, \quad \mathrm{TMR}_{x}=\frac{G_{\leftarrow, \leftarrow}-G_{\leftarrow, \rightarrow}}{G_{\leftarrow, \leftarrow}},
$$

where we denote the positive (negative) $z$ direction as $\uparrow(\downarrow)$ ), and the positive (negative) $x$ direction as $\leftarrow(\rightarrow)$.

Figure 1 shows $\mathrm{TMR}_{z}$ for a symmetric GaMnAs/AlAs/GaMnAs junction as a function of the bulk spin polarization $P$ for two hole densities $p=0.1 \mathrm{~nm}^{-3}$ and $p=0.4 \mathrm{~nm}^{-3}$. The barrier height is $V_{b}=300 \mathrm{meV}$. Both the Julliere expression and $\mathrm{TMR}_{z}$ are increasing functions of the electrode spin polarization $P$. However for intermediate values of the polarization and $p=0.4 \mathrm{~nm}^{-3}$ the difference between the Julliere and the calculated TMR can be of a factor of two. The discrepancy remains if we use the Fermi surface polarization ${ }^{21} P^{F S}$ instead of the bulk polarization $P$. In order to address whether the discrepancy is due to the SO interaction, Fig. 1 also shows $\mathrm{TMR}_{z}$ in the case where the SO interaction is suppressed in the barrier. Interestingly, $\mathrm{TMR}_{z}$ is larger when SO is suppressed and closer to the Julliere result, but still off. tion modifies the conventional relation between TMR and
but still off.
Downloaded 10 Feb 2010 to 161.111.180.191. Redistribution subject to AlP license or copyright; see http://apl.aip.org/apl/copyright.jsp

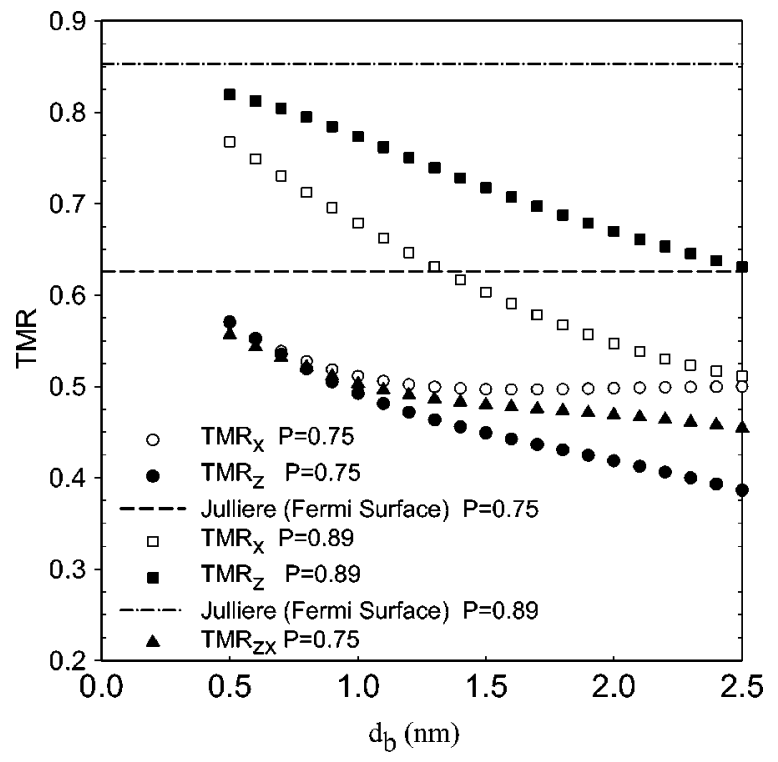

FIG. 2. $\mathrm{TMR}_{z}$ and $\mathrm{TMR}_{x}$ as function of $d_{b}$ for $P=0.75$ and $P=0.89 . V_{b}$ $=300 \mathrm{meV}$ and $p=0.1 \mathrm{~nm}^{-3}$.

Figure 2 shows both $\mathrm{TMR}_{z}$ and $\mathrm{TMR}_{x}$ as a function of the barrier thickness $d_{b}$ for two different values of $P$. The Julliere value, independent of $d_{b}$ is also shown. Due to the strong SO coupling in the system, $\mathrm{TMR}_{z}$ and $\mathrm{TMR}_{x}$ can be quite different, and because of the complicated matching at the interface, its relative magnitude depends strongly on the parameters of the system. In agreement with experiments, and tight-binding calculations ${ }^{1,9}$ we find that TMR decreases rapidly for thin barriers, in marked contrast with Eq. (1). We also plot the TMR when the magnetization is oriented in the [101] direction, $\mathrm{TMR}_{z x}$. This quantity turns out to be almost exactly the average of $\mathrm{TMR}_{x}$ and $\mathrm{TMR}_{z}$, indicating that the experimental ${ }^{9}$ difference in the TMR between the [100] and [110] field directions is due to the SO coupling and not to the cubic magnetocrystalline anisotropy in the GaAs crystal structure.

A quantitative comparison between our results and the experiments is difficult, because quantities like the carrier density and band offset are unknown in the heterostructures. Even $d_{b}$ is not accurately known. ${ }^{9}$ Qualitatively, though, our results account for the decreases of TMR with the barrier thickness, ${ }^{9}$ and also show a strong dependence of the TMR values on the angle formed by the magnetization and the current flow.

Impurity scattering combined with the strong $\mathrm{SO}$ coupling produces anisotropy in the dc transport properties of bulk GaMnAs. ${ }^{22}$ We have analyzed the variation of the ballistic tunneling resistance when the current flow is parallel or perpendicular to the magnetization. In Fig. 3 we plot the anisotropy tunneling magnetoresistance (ATMR):

$$
\operatorname{ATMR}=\frac{G_{\uparrow, \uparrow}-G_{\rightarrow, \rightarrow}}{G_{\uparrow, \uparrow}} .
$$

The tunneling current can change up to $6 \%$ for large values of $P$. This number is comparable with those obtained for bulk. $^{22}$ Therefore, in such a heterostructure, the ATMR can add a significant contribution to the bulk anisotropy magnetoresistance of the electrodes.

In conclusion, the presence of strong spin-orbit interac- 


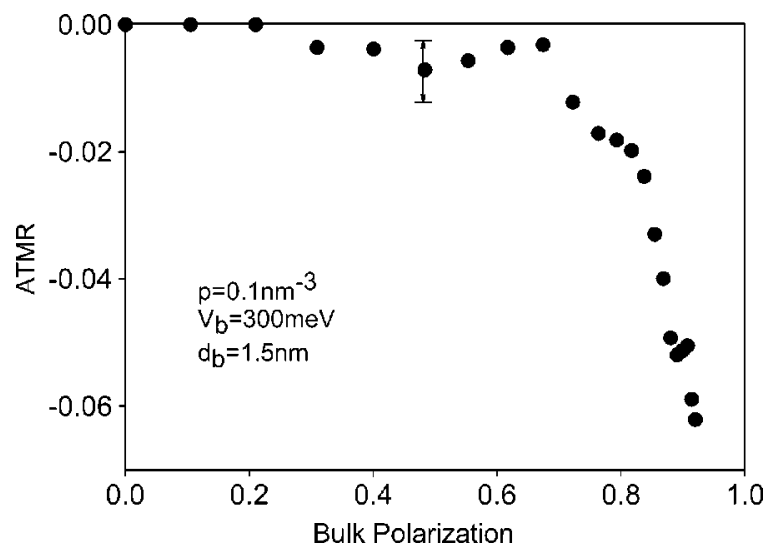

FIG. 3. Anisotropic TMR as function of the bulk polarization. The error bar is an estimation of the numerical error in the calculations.

spin polarization of the electrodes. As in the conventional case, our results show that TMR is an increasing function of the electrodes polarization $P$, quantitatively different than Eq. (1). Large values of TMR can be reached for sufficiently high $P$ even in the presence of strong spin-orbit interaction. In contrast with Eq. (1), TMR depends on the barrier thickness. Therefore, Eq. (1) is not an appropriated tool for inferring the spin polarization of the electrodes in GaMnAs based heterostrucures. Finally, because of the spin-orbit interaction, both the TMR and the conductance depend on the angle between the current and the magnetization orientation. These are qualitatively new physical phenomena that might be exploited to build spin valves with new functionalities.

This work was supported in part by MCYT of Spain under Contract Nos. MAT2002-04429-C03-01, MAT200200139, MAT2003-08109-C02-01, Fundación Ramón Areces,
Ramon y Cajal Program and UE within the Research Training Network COLLECT.

${ }^{1}$ J. S. Moodera and G. Mathon, J. Magn. Magn. Mater. 200, 248 (2000).

${ }^{2}$ P. Grünberg, Phys. Today 54, 31 (2001).

${ }^{3}$ M. Julliere, Phys. Lett. A54, 225 (1975).

${ }^{4}$ J. C. Slonczewski, Phys. Rev. B 39, 6995 (1989).

${ }^{5}$ A. H. MacDonald, T. Jungwirth, and M. Kasner, Phys. Rev. Lett. 81, 705 (1998).

${ }^{6}$ K. Edmonds, K. Wang, R. Campio, A. Neumann, N. Farley, B. Gallagher, and C. Foxon, Appl. Phys. Lett. 81, 4991 (2002).

${ }^{7}$ H. Ohno, D. Chiba, F. Matsukura, T. Omiya, E. Abe, T. Dietl, Y. Ohno, and K. Ohtani, Nature (London) 408, 944 (2000); D. Chiba, M. Yamanouch, F. Matsukura, and H. Ohno, Science 301, 943 (2003).

${ }^{8}$ D. D. Awschalom, D. Loss, and N. Samarth, Semiconductor Spintronics and Quantum Computation (Springer, New York, 2002).

${ }^{9}$ M. Tanaka and Y. Higo, Phys. Rev. Lett. 87, 026602 (2001).

${ }^{10}$ R. Mattana, J.-M. George, H. Jaffres, F. Nguyen Van Dau, A. Fert, B. Lepine, A. Guivarc'h, and G. Jezequel, Phys. Rev. Lett. 90, 166601 (2003).

${ }^{11}$ C. Rster, T. Borzenko, C. Gould, G. Schmidt, L. W. Molenkamp, X. Liu, T. J. Wojtowicz, J. K. Furdyna, Z. G. Yu, and M. E. Flatte, Phys. Rev. Lett. 91, 216602 (2003).

${ }^{12}$ J. G. Braden, J. S. Parker, P. Xiong, S. H. Chun, and N. Samarth, Phys. Rev. Lett. 91, 056602 (2003).

${ }^{13}$ T. Dietl, H. Ohno, F. Matsukura, J. Cibert, and D. Ferrand, Science 287, 1019 (2000).

${ }^{14}$ M. Abolfath, T. Jungwirth, J. Brum, and A. H. MacDonald, Phys. Rev. B 63, 054418 (2001).

${ }^{15}$ J. Fernández-Rossier and L. J. Sham, Phys. Rev. B 64, 235323 (2001).

${ }^{16}$ L. Brey and G. Gómez-Santos, Phys. Rev. B 68, 115206 (2003).

${ }^{17}$ R. Wessel and M. Altarelli, Phys. Rev. B 39, 12802 (1989).

${ }^{18}$ C. Y. Chao and S. L. Chuang, Phys. Rev. B 43, 7027 (1991).

${ }^{19}$ Y. X. Lius, D. Z. Y. Ting, and T. C. McGill, Phys. Rev. B 54, 5675 (1996).

${ }^{20}$ A. G. Petukhov, A. N. Chantis, and D. O. Demchenko, Phys. Rev. Lett. 89, 107205 (2002).

${ }^{21}$ I. I. Mazin, Phys. Rev. Lett. 83, 1427 (1999).

${ }^{22}$ T. Jungwirth, M. Abolfath, J. Sinov, J. Kucera, and A. H. MacDonald, Appl. Phys. Lett. 81, 4029 (2002). 